\title{
Mobile phone data analysis guidelines: applications to monitoring physical distancing and modeling COVID-19
}

\author{
Nishant Kishore ${ }^{1}$, Mathew Kiang ${ }^{2}$, Kenth Engø-Monsen ${ }^{3}$, Navin Vembar $^{6}$, Satchit Balsari ${ }^{4,5}$, \\ Caroline Buckee ${ }^{1}$
}

${ }^{1}$ Center for Communicable Disease Dynamics, Department of Epidemiology, Harvard TH Chan School of Public Health, Boston, USA

${ }^{2}$ Center for Population Health Sciences, Stanford University School of Medicine, Stanford, USA

${ }^{3}$ Telenor Research, Oslo, Norway

${ }^{4}$ Department of Emergency Medicine, Beth Israel Deaconess Medical Center and Harvard Medical School, Boston, USA

${ }^{5}$ India Digital Health Network, Lakshmi Mittal and Family South Asia Institute, Harvard University, Cambridge, USA

${ }^{6}$ Camber Systems, Washington, DC, USA

\begin{abstract}
There has been a recent surge of interest in using mobility data from mobile phones to monitor social distancing and model the spread of SARS-COV-2, the virus that causes COVID-19.

Despite several years of research in this area, standard frameworks for aggregating and making use of different data streams from mobile phones are lacking and difficult to generalize across data providers. Here, we provide a comprehensive set of guidelines for aggregation principles and procedures for different mobile phone data streams, and describe how aggregated data are used in research and policy. We argue that the principles of privacy and data protection are critical in assessing more technical aspects of aggregation, and should be an important central feature guiding partnerships with governments who make use of research products.
\end{abstract}




\section{Introduction}

In response to the COVID-19 outbreak, technology companies and mobile phone operators are coming forward to provide important, epidemiologically-relevant data ${ }^{1,2}$. There are several ways that these data from mobile devices could help to inform policies that reduce the spread of the virus. For example, aggregated location data from mobile phones can be used to provide important population-level information about how people are moving around ${ }^{3-5}$. Given that social distancing interventions - including travel restrictions, working from home, closing schools, universities, and non-essential retail and restaurant venues, and canceling social gatherings - are the only interventions available to slow transmission in the early stage of the epidemic, monitoring how well public messaging is working will be key to its successful implementation ${ }^{6}$.

Aggregated mobility data do not provide information about individual behaviors or contact rates. Rather, they give a description of the population's movements and offer direct estimates of travel connectivity between locations, and this can help inform the risk of importation due to travel from different places, the impact of travel restrictions, and spatial epidemiological models

7,8 . They also provide a general measure of the kinds of travel behavior occurring within a particular population, depending on the spatial resolution of the data. For example, aggregated mobility data can tell you how much people are moving around, how far they go each day, and how many different people visit particular locations ${ }^{9}$. In the dense cities of the global south, such data may help identify the busiest transport or migration routes, where populations may need significant economic support to practice distancing, or a re-think of strategy ${ }^{10,11}$.

Any mobile phone data should be used with a public health purpose in mind during this crisis, and privacy and ethical considerations are critical ${ }^{12}$. Each country has national regulators that provide guidance on exactly how mobile phone data can be used, and the legal frameworks involved will vary, particularly in the context of a public health emergency ${ }^{13,14}$. In Europe, for example, most national laws provide that location data can only be used by the operator when they are made anonymous, or with the consent of the individual ${ }^{12,15,16}$. For aggregated data the risks are much reduced, but in general, the benefits of using personal data must outweigh the risks to privacy and liberty. Here we outline considerations for the use of these data for public health purposes, specifically in the context of monitoring social distancing interventions and modeling the spread of COVID-19. 


\section{General considerations for public health use}

We distinguish between two main types of data based on how they are collected. The first is mobile operator data, which is collected routinely in the form of either Call Detail Records (CDRs) or a continuous stream of network signaling data. CDRs provide a cell tower ID for calls, texts, or other uses associated with a SIM card, whereas network signaling data give continuous information about the cell tower that a handset is connected to as long as it is on. In both cases, the location of the cell tower provides an approximate location for the user at the time of the call or text, somewhere on the order of $1 \mathrm{~km}-5 \mathrm{~km}$ grid square spatial resolution, depending on the density of cell towers in that region. Given the near universal penetration of phones, the primary advantage of CDR data is that they are much more representative than data from applications (apps) in places where many people do not have smartphones ${ }^{17,18}$.

The second type of data are GPS traces that are collected from different social media and other apps. GPS trace data provide insights into the precise location (down to a $\sim 5-20 \mathrm{~m}$ resolution) of the device over time. These data are however more limited in terms of coverage and representativeness, particularly in low- and middle-income countries (LMICs), but can be more granular in some cases ${ }^{19}$. Both types of data must be aggregated in time and space in order to comply with privacy principles that ensure the individual using the device cannot be identified, even indirectly, from the data. Public health emergencies do not provide a blanket excuse to abandon all privacy protections.

Spatial and temporal aggregation: In each case, the data will ultimately be aggregated in space and time to correspond to a useful administrative or other spatial boundary, and on a time scale that can provide relevant information to decision makers receiving epidemiological information or providing public messaging - possibly every day given the rapidly changing landscape of the COVID-19 outbreak. Administrative boundaries are often most helpful for policy makers, and the necessary scale will depend on whether the location in question is rural or urban; cities sometimes need smaller spatial scales.

In every case, the spatial aggregation must be checked to ensure that the numbers of unique devices do not fall below thresholds that enable re-identification of individuals or groups from the data. Allowing counts of one, or just hashing identifiers in a dataset, risks easy re-identification of individuals ${ }^{20,21}$. There is currently no universal agreement on what a robust threshold should be, but in Europe, mobile operators are using thresholds ranging from 20 to 5, Facebook Data for Good ${ }^{22}$ Geoinsights portal typically sets the threshold at 10 users $^{23}$ and Camber Systems, another data provider using Advertisement Technology (ad-tech) data, uses 20. This means that counts of people that fall below these thresholds are censored from the dataset and will not be included in the shared data. There are additional technical ways to limit the possibility of re-identification, but these numbers provide at least a rule of thumb ${ }^{24}$. Other approaches to preserving privacy include adding noise to aggregated data products, aggregating after applying differential privacy with appropriate privacy budgets, or resetting the cohort of analysis daily ${ }^{20,25-27}$. 
Representativeness: Data providers must be clear about the representativeness of their data, while respecting privacy, and provide aggregated information about the resulting uncertainty in their estimates. There are three important considerations here:

- market share: what fraction of people overall do these data represent?

- demographic representativeness: who are the people generating the data, with respect to age groups, sex, and socioeconomic status, compared to the overall population? (This is important so the biases are clear - sometimes, the most vulnerable populations are excluded and datasets do not usually include children).

- geographic representativeness: generally, these data will be most representative of urban populations, and understanding how well they represent the rural part of the population is important to communicate to users of aggregated data.

The rapid growth in data brokers seeking to make such data available for COVID-19 epidemiological modelling presents an opportunity to begin to standardize uncertainty estimates. One possible strategy for providing comparable metrics of evaluation is to use a formal metric for representativeness ${ }^{28}$. While asking for specific distributions of a population's demographic characteristics by region may not be feasible, an L1 normalization across all demographic metrics of interest standardized to the same comparison (census data for example), would allow for users to evaluate representativeness between data sources.

Ideally, data will be normalized and compared to a gold standard such as recent census data, or at least cross-validated against similar data sets. Normalization is often challenging, so the more information (demographics, socio-economic status) that is known about the kinds of subscribers a particular provider has in general, the more confident we can be in the representativeness of the data. Although it is not routinely done, clear communication and quantification of uncertainty to any users of aggregated outputs is critical to prevent misinterpretation or misplaced confidence in mobility estimates.

Importantly, for privacy and ethical reasons, we do not endorse disaggregation of information by demographic or other status, unless it is coarse enough to prevent the possible misuse of the information. For example, while it may be reasonable under some circumstances to disaggregate data based on $>60$ years old versus $<60$ years old, it would not be appropriate to disaggregate by ethnicity, sex, or other groupings that could lead to discrimination.

Managing outliers: Outliers should be discarded; these may be devices that are calling an unreasonable number of times in a day, or not calling at all for weeks at a time, for example. It is very likely that some of these devices are machines, and as such, it is plausible that they do not represent human behavior. The goal is to discard signals that are not consistent with the movements we are trying to examine: personal mobility patterns. The exact parameters for discarding outliers will depend on the population and the operator, but data science teams within operators and companies generally have a good grasp of what is and is not likely to be 
representative. Again, iterative communication between operators and researchers or the users of output is critical on these issues.

Establishing baselines: An important component of almost all analyses will be a baseline against which to compare changes in travel, and in many cases, a "home" location for particular devices, against which travel can be measured. Often, mobility data provided to public health practitioners are most useful if they are given as relative change, in percent, against a baseline over time, rather than exact numbers. Here, baselines can be established by making sure that data analysis reaches back in time before the outbreak started and social distancing interventions were put in place. Seasonal mobility patterns are important, so if it is possible to compare data to the same month in a previous year, that is ideal, but most companies do not store data for such a long time period due to data retention policies ${ }^{29-31}$. Clear communication of the baseline is critical, and the uncertainty associated with it, so that decision makers can make sense of the changes they see in mobility. Currently, the Facebook Data for Good Geoinsights platform establishes their baselines by using data for the 45 days preceding the first date of daily data collection.

Data sharing: The needs of policy makers will be different from the needs of groups that want to model the spread of the outbreak. Operators are beholden to national regulators; these are generally independent bodies that determine what CDRs can be used for. In each case, approval from the regulator is needed to conduct these aggregations and share them with researchers or policy makers. Often, this is the most lengthy step in the process, and discussions with the regulator should be started early. GPS trace data from social media applications or ad-tech companies are often collected by data aggregators for commercial purposes. These entities often have existing pipelines for collection and validation of these data sources and may even be able to provide insight into representativeness and methods for standardization to the general population in a region. Partnership with data aggregators may simplify processes for data use or licensing agreements, reduce the number of data sources which feed into local analytic pipelines, and reduce exposure to granular GPS trace data. For example Camber Systems aggregates and pre-processes data and provides that to researchers, rather than the raw trace data.

In the case of sharing aggregated data with researchers, data use agreements and compliance with university ethics processes are essential prerequisites. Once met, onboarding documentation should be provided to allow researchers to access aggregated, privacy-optimized data. While many platforms can be used to provide these data, the priority should be ease and automation of data downloads into analytic pipelines. For example, data may be provided using a restricted set of queries to a researcher-facing database through a structured API and individual access keys. In every case, we recommend adherence to the highest privacy frameworks and agreement by all parties on these principles up front. 


\section{Reporting and scaling:}

Generally, policy makers need information that provides situational awareness and a way to critically evaluate different strategies, including modeled outcomes. Teams within government that produce these insights may want simplified metrics that they can compare across different data sets, including rates of change in travel in different locations and between different places, compared to the previous week or relative to a particular intervention. This can be provided in the form of heat maps, as simple line graphs, or as a table. In contrast, researchers need to parameterize models, so questions of normalization, representativeness, and more complex travel patterns may be very important.

Data needs, the capacity to analyze data, and the utility of certain metrics will vary by spatial resolution. For example, city administrators may be interested in identifying hotspots of conglomeration, or patterns in certain types of activity (visits to grocery stores, transit hubs, schools), while state governments may be more concerned with travel networks across administrative boundaries. If providers are able to dedicate resources in response to a crisis, they might consider generating analyses at varying spatial resolutions specific to each use case. In large and quickly moving crises, however, the number of requests for such data can scale up much more quickly than provider specific capacity to respond. In this case it is important that providers generate standard pipelines which provide metrics aggregated to the most granular spatial resolution possible, and empower researchers to, in turn, provide location specific analyses. Context is key, and a one-size-fits-all product is not likely to work. Offering a platform that provides access to multiple researchers, will allow the use and application of these critically needed data to scale at the rate currently needed. National policies can regulate who may be provided such access. 


\section{Strategies for data aggregation across space and time}

Temporal aggregations

Although many different kinds of aggregation are possible, a straightforward decision would be to aggregate first on a daily basis. Sub-daily aggregations must focus on providing useful windows of time which capture mobility relevant to the local population such as windows which capture hours of sleep, daytime work mobility and evening leisure mobility. For both CDR and GPS trace data, it may be tempting to ease the burden of computation by aggregating to Coordinated Universal Time (UTC), however it is important to standardize the temporal binning to occur for the local time zone. While this may be complicated for spatial regions which encompass more than one time zone, it ensures that the windows of temporal aggregation are relevant locally.

For GPS traces, aggregation steps must adhere to differential privacy standards as close to the source (the individual device providing the data) as possible ${ }^{26,32,33}$. In many cases, manufacturers are already implementing these as the standard to ensure that data are transformed before they leave the device ${ }^{27,34,35}$. These manufacturer standards, however, are not ubiquitous and device types vary greatly by country and socio-economic status leading to heterogeneity in privacy protection provided by these measures.

Spatial aggregations: CDRs versus GPS traces

For CDRs, aggregations must first occur at the cell tower level, and be executed by the operator's data science teams. Cell tower data can then be aggregated up to the appropriate spatial scale, generally a grid or administrative unit.

For the GPS trace data there can essentially be an infinite number of locations, and one of the first decisions will be the spatial units of analysis; as with CDRs, gridded cells or administrative units are both reasonable. Once that decision has been made, the considerations are essentially the same as for operator data above.

While data can be aggregated and provided at the most granular spatial scale feasible which ensures data privacy, we advocate for sharing at the lowest spatial resolution (i.e. least granular) required to answer a particular, policy-relevant question. Granularity will vary greatly by administrative areas and population density, with similar considerations as for CDRs; namely, in cities the resolution needed (e.g. neighborhood level) may be higher than in rural areas $(1 \mathrm{~km}$ $x 1 \mathrm{~km}$ grid squares may be sufficient). Providers may consider simply dropping regions or tiles which don't meet a certain threshold of users contributing data or automatically upscaling the resolution regions with low population density. Outputs should include a numerically complete subset of lower resolution spatial scales. For example, if admin region $A$ is composed of 4 subregions, then the sum of the population in each subregion and the metrics calculated for each subregion should be readily rescaled to represent all of admin region A either through a simple sum or a weighted process. 
Finally, spatial data should be provided with enough information for researchers to be able to map it to their region of interest. This includes geometry of all transition vectors and spatial information for all delineated regions. There are context-specific considerations here, and geographic delineations of administrative boundaries used by policy makers will vary. 


\section{Mobility metrics and their relation to social distancing and COVID-19 response}

Here we outline the metrics that we have identified as useful for observing population mobility under different types of social distancing interventions, and as inputs for mechanistic models of disease spread. The transition between regions will generally be used as a proxy for a migration rate between locations, as part of a meta-population model. The measures below that describe within-area movement patterns are more useful as a proxy for possible changes in the contact rate within populations, although the precise mapping of metrics to a contact rate parameter is not well-defined, and will depend on multiple population-specific factors including household structures, population density, cultural and economic parameters, and the policies being implemented. Nearly all metrics calculated by CDR can be calculated with GPS traces, therefore, we have separated the metrics into those that can be calculated by both data sources and those which are exclusive to GPS trace data.

\section{Definitions}

The first step in calculating any of these mobility metrics is defining the:

1) Population: a description of the unit of measurement that contributes data to the analysis such as unique users or unique devices. Here these are designated as $i$ and $j$.

2) Spatial granularity of categorization: a description of the size of the user locations that are used for categorization. For example, unique locations that are visited by a user may be defined as tile grids, tower catchment areas or GPS radii from points of interest for internal analysis. Here these are designated as $a$ and $b$.

3) Spatial granularity of aggregation: a description of the size of the regions of interest for which data are aggregated before being shared. For example, the metrics calculated for the population can be aggregated across all users in a region of interest such as neighborhood or county. Here these are designated as $A$ and $B$.

4) Temporal granularity of categorization: a description of the time bins that are used to categorize user location. For example, the top location that a user logs data in every hour. These are designated as $t$.

5) Temporal granularity of aggregation: a description of the period of time for which data are aggregated for all users $i$ in region $A$. For example we might be interested in the average numbers of locations $a$ (defined at top location per $t$ minute bin) over all space visited by users $i$ in region $A$ over the course of an 8 hours window. Here these are designated at $T$.

6) Temporal thresholds: rather than calculating locations by top location $a$ in each time bin $t$, providers may decide to calculate locations $a$ as those where the user $i$ spends at least a certain amount of time. This threshold is then designated at $T^{*}$.

Using these definitions, a provider may generate for each individual $i$ a set of locations at spatial scale $a$ for each time bin $t$ in the time window $T$. The values for $a$ can be directly mapped to larger regions of aggregation $A$. For example, for a given user, you can calculate their location as defined by presence in a $600 \mathrm{~m} \times 600 \mathrm{~m}$ Bing Tile ( $a$ : zoom level $16^{36}$ ) for every 
30 minute segment $(t)$ over the course of 24 hours $(T)$. Each bing tile is also mapped onto a county $A$ for which all of the data are aggregated. We define this set of time specific locations as $M_{i T}=\left\{a_{i t 1}, a_{i t 2}, \ldots, a_{i t n}\right\}$ where $a_{i t n}$ refers to a specific bin $t$ in time window $T$. This set, $M_{i T}$ can then be manipulated in many ways including calculation of unique or top locations, conditional on spatiotemporal categorization and aggregation. Not all users will provide enough information to generate a full set $M_{i T}$, therefore, the provider should be transparent about any interpolation or imputation steps taken to make the set more robust.

Due to differences in spatiotemporal resolution, the definition of a stay location $a$ will differ between CDR and GPS. For CDR, a stay location represents the tower, grid, or administrative region where a user's phone was located. For GPS, depending on the spatiotemporal resolution, some preprocessing may occur. Typically, we can define a stay location as an area of size $a$ where all movement occurred for at least $T^{*}$ time units (e.g., a circle with a radius of $25 \mathrm{~m}$ where all movement remained inside for at least 30 minutes). ${ }^{24}$ Different thresholds $T^{*}$ can be used (e.g., 5 minutes, 1 hour, etc.) and the proper threshold will depend on both technological and computational constraints and the relevant question.

These key values may change depending on the metric due to privacy-preserving or computational limitations, however, the choice and rationale of these values must always be clearly communicated.

\section{Both CDR and GPS trace metrics}

Population distribution and dynamics: For most metadata and for all modeling work, an estimate of the population in a particular region at a particular time point is going to be essential as a denominator. This is the number of unique SIMs (CDR) or device IDs (GPS) that spend most of their time in a given area. This measure should be compared to census data and other sources of information to normalize the estimates and quantify uncertainty or potential bias. To estimate this quantity, we typically assign each user to a "home" location. This is often either the most frequent location or the area where the user spends their most time in the set of locations $M_{i T}$. In the case of continuous GPS data collection, one can also use location at midnight local time, or a range around that time if data availability is not continuous. The sum of unique users in each region $A$ is then used as the population estimate for that time window $T$. This value is denoted as

$$
N_{A T}=\sum_{i} x_{i A T}
$$

where $x_{i A T}=1$ if the mode of $M_{i T}$ for time $T$ is in $A$ and 0 otherwise.

Number of significant locations: The average number of "significant locations" provides an indication of how many distinct places users spend a substantial amount of time. Normal human 
mobility involves very few significant locations - usually, home, work, or school. However, different types of "shelter-in-place" orders may result in different types of behaviors. For example, strict "never leave home" orders would result in a reduction of significant locations to 1 while less strict orders may result in increased numbers of local significant locations as individuals attempt to briefly leave their homes.

To calculate the average number of significant locations, we use the set of time varying locations for each user $i, M_{i T}$ as described above. Additional metadata such as duration spent at each location or time of arrival may also be important. Once the set of significant locations for each user has been estimated, the average across a region, grid, or other area of interest can be estimated as the sum of total unique locations visited by user $i$ whose home location is in region $A$ divided by the total number of users whose home location is in region $A$.

$$
\overline{M_{A T}}=\frac{\sum_{i \in A} \operatorname{card}\left(M_{i T}\right)}{N_{A T}}
$$

Transition between regions: This value provides an estimate of mobility between locations, which can be used in models to estimate the spatial spread of the virus. Transition matrices should include number, index, or proportion of unique users or SIMs who move from region $A$ to region $B$. Users should only contribute to the transition matrices once within each window of time to ensure that the numbers represent users and not "trips" between regions. As the window of time considered decreases, researchers will be better able to understand within day heterogeneity in movement between regions. This metric is generally captured by evaluating the location at which a user spends the most time during the beginning of the window, if the user moves to another region, and the location that the user is in when they stop moving. If the user visits many locations this captures the destination where the user spends the most time. These data are then aggregated within the spatial and temporal windows and provided as a vector of transition between regions. These values can be used to calculate the percent change in total number of trips which occur between and within regions of interest providing metrics of internal and external mobility. It is important to consider that these metrics will vary greatly with the spatial scale of the region and the time window considered. We recommend that $T$ is at most 4 hours and suggests that decisions on spatial granularity of categorization and aggregation are made in collaboration with the end users of these data.

This can be calculated by splitting the time window $T$ into two halves ( $T 1$ and $T 2$ ). Then simply calculate the mode for each set $M_{i T 1}$ and $M_{i T 2}$ resulting in $a_{i T 1}$ and $a_{i T 2}$. Here we assume that for some time window $T$ the user transitioned from the modal location in $T 1$ to the modal location in $T 2$. If the user did not transition between locations, the matrix will include these counts on the diagonal. These can then be summed across all users with transitions that do not meet a minimum threshold being dropped. To aggregate to a larger spatial resolution, map $a$ to its corresponding $A$ and sum the transition values for unique pairs of $A$ and $B$. 
Distances traveled: This metric measures the amount of movement occurring within a population. These are calculated for each of the start and stop points of the vectors of travel for both CDRs and GPS traces. It is important to note that for CDRs these will be constrained by tower transitions, and suffer from the problems where two towers may route a call if a subscriber is between them and be upwardly biased in their estimates. As such, these should not be interpreted as movement patterns. For GPS traces, distance traveled can be simple Euclidean or haversine (spherical) distances computed between points of the trail. In cases where car travel is obvious, routing engines can be used to compute on-ground transit distances. In order to make the traces useful, depending on the data source, care should be taken to remove points that represent impossible travel (accounting for different modes of transportation), data that may be synthesized and thus not represent reality, and to avoid data skew created by individual devices putting out significantly different number of points, potentially again with synthesized data ${ }^{37}$. Average and total distances of these vectors are then weighted by the number of users who made the transition. This provides a total or average distance moved by users in a given region.

$$
\overline{D_{i}}=\sum_{l=2}^{n}\left|a_{i l}-a_{i l-1}\right|
$$

where $\left|a_{i l}-a_{i l-1}\right|$ is the distance between location $a_{i l}$ and $a_{i l-1}$. Another option would be to directly provide the distances between these locations as a value for each pair of locations, allowing researchers to aggregate and calculate as they see fit.

Radius of Gyration (RoG): This is a measure that can handle some missing data and gives a general sense of the extent of individual travel by incorporating both the number of trips as well as the distance of each trip ${ }^{20-22}$. To compute the RoG for user $i$, first calculate the root mean squared distance of a user's movement across space over a given window of time from their center of gravity.

$$
r_{g}(i)=\sqrt{\frac{1}{n} \sum_{l=1}^{n}\left|a_{i l}-\bar{a}\right|^{2}}
$$

where

$$
\bar{a}=\frac{1}{n} \sum_{l=1}^{n} a_{i l}
$$


is the center of gravity. (Note, that the center of gravity is not necessarily the same as the "home" location defined above. For example, in the extreme case of all trips being in one direction.) Then for each user, generate their home region $A$ as the region in which they spend the most time in their location set $M_{i T}$. Then aggregate this value across a population in a given region and provide an average and percentiles.

Regularity of movement: In general, human mobility is highly predictable ${ }^{9,38}$. This predictability has great importance for urban planning, traffic forecasting, and public health. A formal measure of (un)predictability is location entropy ${ }^{25,26}$. Intuitively, a low location entropy means an individual's time spent at their significant locations is highly predictable. Conversely, high location entropy suggests predicting an individual's location is difficult. Therefore the lowest location entropy would be achieved by a user who spends the exact same amount of time in the same places in every time window. Similar to the measure of significant locations, the interpretation of this metric depends on the "strictness" of the stay at home order. It is important to note that the distance between locations is not taken into account here.

Using the set of locations defined above, $M_{i T}$, the random entropy (or uncertainty) of user $i$ can be calculated as $S_{i}^{r a n d}=\log _{2} L_{i}$ where $L_{i}$ is the number of distinct locations in $M_{i T}$. This measure assumes a person's location is uniformly distributed among all $L_{i}$ observed distinct locations in $M_{i T}$. The uncorrelated Shannon entropy of the user $i$ is

$$
S_{i}^{u n c}=-\sum_{k=1}^{L_{i}} p_{k} \log _{2} p_{k}
$$

where $p_{k}$ is the frequency of the users visit to their $k^{t h}$ location. Other, most sophisticated, measures of location entropy have also been found to describe movement predictability well ${ }^{9}$.

\section{GPS trace metrics}

Average co-location with individuals in other regions: This value provides an indication of how much contact individuals from one region have with individuals from other regions. This analysis is limited to GPS trace data but provides the most direct measure of contact between different populations. This metric is recalculated and independent for every time window $T$. It is important that users who form the population for this metric meet a minimum threshold for data contributed during time $T$. First, for each user, calculate their set of locations for each time segment $t$ in time window $T$ (ex: $M_{i T}=\left\{a_{i t 1}, a_{i t 2}, \ldots, a_{i t n}\right\}$ ). Depending on the completeness of the GPS traces, interpolation or imputation of user locations may need to be considered. In this case it is important to clearly state the process for such imputation. Second calculate each user's home region $A$ as the region in which the user spends most of their time at night in a time period $T$. For situations where comparing two different regions $A$ and $B$, calculate the probability of co-location as: 


$$
\frac{\sum_{j \in B} \sum_{i \in A} y_{i j}}{N_{A T} * N_{B T} * \frac{T}{t}}
$$

where

$$
y_{i j}=\sum_{l=1}^{n}\left\{a_{i t l}=a_{j t l}\right\}
$$

Describes the total number of co-locations in a region of size $a$ that users $i$ and $j$ have over the course of $T . N_{A T}$ is the total population of users whose home location is in region $A$ for time $T, N_{B T}$ is the total population of users whose home location is in region $B$ for time $T$ and $T$

$\frac{T}{t}$ is the total number of time bins $t$ that exist in time window $T$. For situations where comparing the same region, calculate the probability of co-location as:

$$
\frac{\frac{\sum_{j \neq i \in A} \sum_{i \in A} y_{i j}}{2}}{N_{A T} *\left(\frac{N_{A T}-1}{2}\right) * \frac{T}{t}}
$$

This method has been led and implemented by the team at Facebook's Data for Good team and provides direct measures of probability of contact between different regions in their population. For their metric they define $a$ as Bing tiles at zoom level 16, $A$ as a county in the USA or an admin level 3 equivalent spatial area, $t$ as 5 minutes and $T$ as a week. Unlike other metrics provided by Facebook, this one is independent for each time window and therefore doesn't have a baseline. In countries without admin level 3 information this $\mathrm{i}$

Measures of "staying put": This is a direct measure of how much time people are spending in one location as opposed to moving around and is relevant to measuring the impact of shelter in place policies and other strict lockdowns. This should be inversely related to the measures of mobility above (average distance traveled and radius of gyration). To calculate this measure, generate the full set of unique locations $M_{i T}$ for each user $i$ for a given time window $T$. Then assign each user to a home region $A$ as the region $a_{i T}$ where the user spends the most time in a time window $T$. Finally, count the number of unique locations for each user $i$ in region $A$ and calculate the proportion of all users $i$ in region $A$ who reported only one unique location during the time window $T$.

Measures of travel to geofenced locations: This will provide an indication of the nature of the travel that is being undertaken ${ }^{1}$. First, define a set of locations of size $a$ in region $A$ which are categorized as being locations of interest. These may include but are not limited to parks, commercial areas or grocery stores. These locations may be grouped together in categories, or 
specify different points of interest. To calculate this metric, first, generate the full set of unique locations $M_{i T}$ for each user $i$ in a time window $T$. Second assign each user to a region $A$ based on the location $a_{i t}$ that the user $i$ spent the most amount of time in. Finally, for all users $i$ in region $A$, calculate the proportion that visited a geofenced location in a time period $T$.

Figure 1: Examples of geofencing for locations of interest.

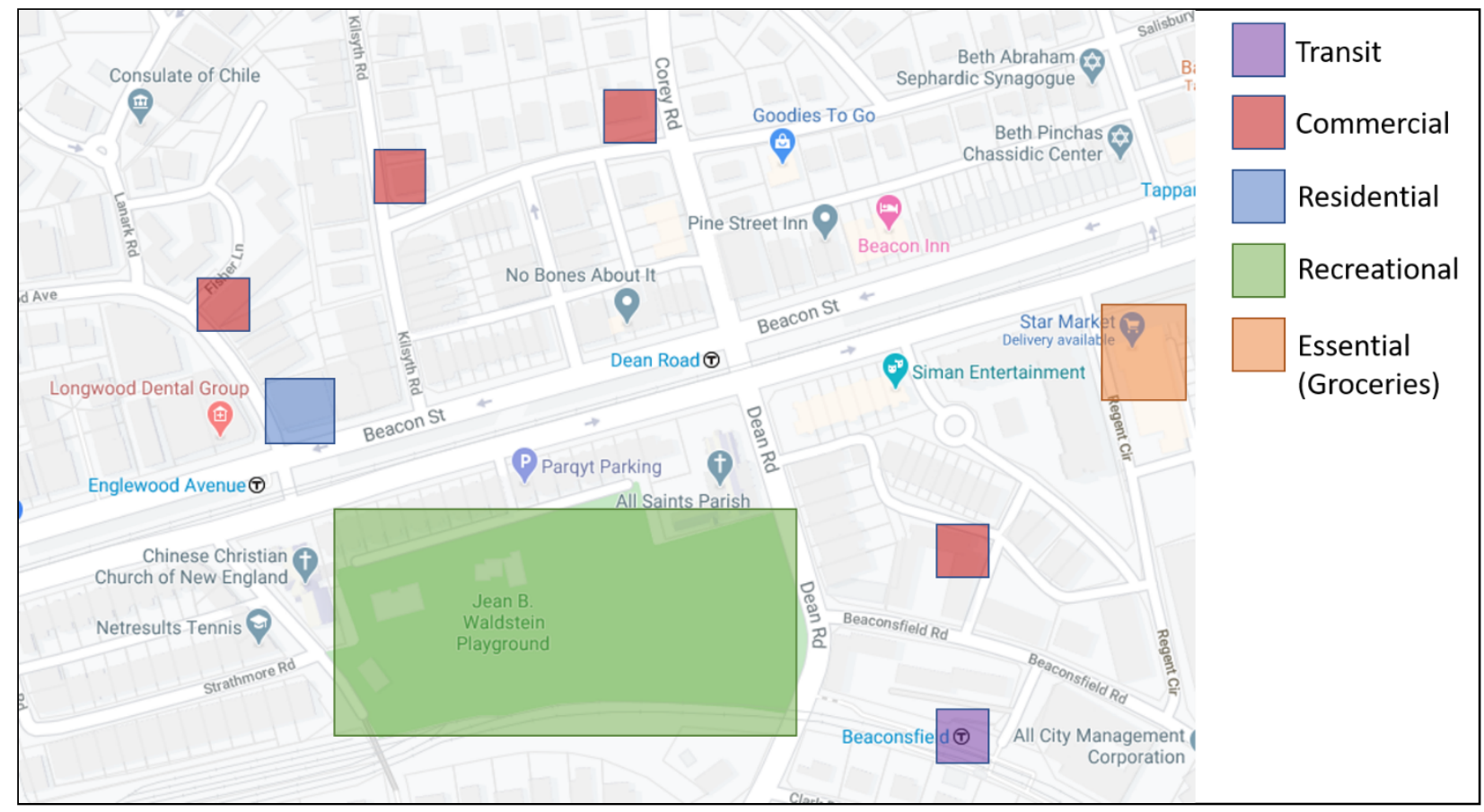




\section{Conclusions}

Together, these metrics and data considerations may provide insights for policy makers, and could inform epidemiological models, about social distancing and the spatial spread of COVID-19. They are likely to play an important role in planning rollbacks as they will help policy makers and epidemiologists estimate the impact on various relaxations on actual mobility patterns on the ground. 


\section{References}

1. Helping public health officials combat COVID-19. Google. https://blog.google/technology/health/covid-19-community-mobility-reports/. Published April 3, 2020. Accessed April 12, 2020.

2. Facebook Data for Good. Facebook Disaster Maps: Methodology. Facebook Res. June 2017. https://research.fb.com/facebook-disaster-maps-methodology/. Accessed May 14, 2019.

3. Palmer JRB, Espenshade TJ, Bartumeus F, Chung CY, Ozgencil NE, Li K. New Approaches to Human Mobility: Using Mobile Phones for Demographic Research. Demography. 2013;50(3). doi:10.1007/s13524-012-0175-z

4. Wesolowski A, Buckee CO, Engø-Monsen K, Metcalf CJE. Connecting Mobility to Infectious Diseases: The Promise and Limits of Mobile Phone Data. J Infect Dis. 2016;214(suppl_4):S414-S420. doi:10.1093/infdis/jiw273

5. Williams NE, Thomas TA, Dunbar M, Eagle N, Dobra A. Measures of Human Mobility Using Mobile Phone Records Enhanced with GIS Data. PLOS ONE. 2015;10(7):e0133630. doi:10.1371/journal.pone.0133630

6. Nonpharmaceutical Interventions for Pandemic Influenza, National and Community Measures. Emerg Infect Dis. 2006;12(1):88-94. doi:10.3201/eid1201.051371

7. Wesolowski A, Eagle N, Tatem AJ, et al. Quantifying the Impact of Human Mobility on Malaria. Science. 2012;338(6104):267-270. doi:10.1126/science.1223467

8. Wesolowski A, Qureshi T, Boni MF, et al. Impact of human mobility on the emergence of dengue epidemics in Pakistan. Proc Natl Acad Sci. 2015;112(38):11887-11892. doi:10.1073/pnas.1504964112

9. Lu X, Wetter E, Bharti N, Tatem AJ, Bengtsson L. Approaching the Limit of Predictability in Human Mobility. Sci Rep. 2013;3(1):1-9. doi:10.1038/srep02923

10. Althouse BM, Scarpino SV, Meyers LA, et al. Enhancing disease surveillance with novel data streams: challenges and opportunities. EPJ Data Sci. 2015;4(1):1-8. doi:10.1140/epjds/s13688-015-0054-0

11. Wesolowski A, O’Meara WP, Eagle N, Tatem AJ, Buckee CO. Evaluating Spatial Interaction Models for Regional Mobility in Sub-Saharan Africa. PLoS Comput Biol. 2015;11(7). doi:10.1371/journal.pcbi.1004267

12. Buckee C, Engø-Monsen K. Mobile Phone Data for Public Health: Towards Data-Sharing Solutions That Protect Individual Privacy and National Security.; 2016. doi:10.13140/RG.2.1.5027.5440

13. Israel passes emergency law to use mobile data for COVID-19 contact tracing.

TechCrunch. https://social.techcrunch.com/2020/03/18/israel-passes-emergency-law-to-use-mobile-data -for-covid-19-contact-tracing/. Accessed April 2, 2020.

14. Kim MS. South Korea is watching quarantined citizens with a smartphone app. MIT Technology Review. https://www.technologyreview.com/s/615329/coronavirus-south-korea-smartphone-app-qu arantine/. Accessed April 2, 2020.

15. de Montjoye Y-A, Hidalgo CA, Verleysen M, Blondel VD. Unique in the Crowd: The privacy bounds of human mobility. Sci Rep. 2013;3(1):1-5. doi:10.1038/srep01376

16. Rocher L, Hendrickx JM, de Montjoye Y-A. Estimating the success of re-identifications in incomplete datasets using generative models. Nat Commun. 2019;10(1):1-9. 
doi:10.1038/s41467-019-10933-3

17. Zhao Z, Shaw S-L, Xu Y, Lu F, Chen J, Yin L. Understanding the bias of call detail records in human mobility research. Int J Geogr Inf Sci. 2016;30(9):1738-1762.

doi:10.1080/13658816.2015.1137298

18. Wesolowski A, Eagle N, Noor AM, Snow RW, Buckee CO. The impact of biases in mobile phone ownership on estimates of human mobility. $J R$ Soc Interface.

2013;10(81):20120986. doi:10.1098/rsif.2012.0986

19. Alessandretti L, Sapiezynski P, Lehmann S, Baronchelli A. Multi-scale spatio-temporal analysis of human mobility. PLoS ONE. 2017;12(2). doi:10.1371/journal.pone.0171686

20. Sweeney L. Simple Demographics Often Identify People Uniquely. January 2000. doi:10.1184/R1/6625769.v1

21. Narayanan A, Shmatikov V. Robust De-anonymization of Large Sparse Datasets. In: 2008 IEEE Symposium on Security and Privacy (Sp 2008). Oakland, CA, USA: IEEE; 2008:111-125. doi:10.1109/SP.2008.33

22. Facebook Data for Good. Facebook Data for Good. https://dataforgood.fb.com/. Accessed April 16, 2020.

23. Maas P. Facebook Disaster Maps: Aggregate Insights for Crisis Response \& Recovery. In: Proceedings of the 25th ACM SIGKDD International Conference on Knowledge Discovery \& Data Mining - KDD '19. Anchorage, AK, USA: ACM Press; 2019:3173-3173. doi:10.1145/3292500.3340412

24. Montjoye Y-A de, Gambs S, Blondel V, et al. On the privacy-conscientious use of mobile phone data. Sci Data. 2018;5. doi:10.1038/sdata.2018.286

25. Ding Z, Wang Y, Wang G, Zhang D, Kifer D. Detecting Violations of Differential Privacy. In: Proceedings of the 2018 ACM SIGSAC Conference on Computer and Communications Security. CCS '18. New York, NY, USA: ACM; 2018:475-489. doi:10.1145/3243734.3243818

26. Differential Privacy Models for Location-Based Services | Transactions on Data Privacy. https://dl.acm.org/doi/10.5555/2993210.2993212. Accessed April 2, 2020.

27. Andrés ME, Bordenabe NE, Chatzikokolakis K, Palamidessi C. Geo-Indistinguishability: Differential Privacy for Location-Based Systems. Proc 2013 ACM SIGSAC Conf Comput Commun Secur - CCS 13. 2013:901-914. doi:10.1145/2508859.2516735

28. lacus SM, King G, Porro G. Multivariate Matching Methods That Are Monotonic Imbalance Bounding. J Am Stat Assoc. 2011;106(493):345-361. doi:10.1198/jasa.2011.tm09599

29. Wesolowski A, Metcalf CJE, Eagle N, et al. Quantifying seasonal population fluxes driving rubella transmission dynamics using mobile phone data. Proc Natl Acad Sci. 2015;112(35):11114-11119. doi:10.1073/pnas.1423542112

30. Wesolowski A, zu Erbach-Schoenberg E, Tatem AJ, et al. Multinational patterns of seasonal asymmetry in human movement influence infectious disease dynamics. Nat Commun. 2017;8(1):1-9. doi:10.1038/s41467-017-02064-4

31. Lai S, Ruktanonchai NW, Zhou L, et al. Effect of Non-Pharmaceutical Interventions for Containing the COVID-19 Outbreak. https://www.worldpop.org/events/COVID_NPI. Accessed April 5, 2020.

32. Xiong P, Zhu T, Pan L, Niu W, Li G. Privacy Preserving in Location Data Release: A Differential Privacy Approach. In: Pham D-N, Park S-B, eds. PRICAI 2014: Trends in Artificial Intelligence. Lecture Notes in Computer Science. Cham: Springer International Publishing; 2014:183-195. doi:10.1007/978-3-319-13560-1_15

33. Xiao Y, Xiong L. Protecting Locations with Differential Privacy Under Temporal Correlations. In: Proceedings of the 22Nd ACM SIGSAC Conference on Computer and 
Communications Security. CCS '15. New York, NY, USA: ACM; 2015:1298-1309. doi:10.1145/2810103.2813640

34. Apple Differential Privacy Overview. https://www.apple.com/privacy/docs/Differential_Privacy_Overview.pdf. Accessed April 2, 2020.

35. Statt N. Google is open-sourcing a tool for data scientists to help protect private information. The Verge.

https://www.theverge.com/2019/9/5/20850465/google-differential-privacy-open-source-tool -privacy-data-sharing. Published September 5, 2019. Accessed April 2, 2020.

36. Brundritt R, Cai S, French C. Bing Maps Tile System - Bing Maps. https://docs.microsoft.com/en-us/bingmaps/articles/bing-maps-tile-system. Accessed April 8, 2020.

37. Rhee I, Shin M, Hong S, Lee K, Kim SJ, Chong S. On the Levy-Walk Nature of Human Mobility. IEEEACM Trans Netw. 2011;19(3):630-643. doi:10.1109/TNET.2011.2120618

38. Song C, Qu Z, Blumm N, Barabási A-L. Limits of Predictability in Human Mobility. Science. 2010;327(5968):1018-1021. doi:10.1126/science.1177170 